\title{
Prevalence and Clinical Presentations of Dilated Cardiomyopathy in Sudanese Patients with Heart Failure
}

\author{
Suha Mahir Atabani ${ }^{1}$, Siddig Ibrahim Khali ${ }^{1}$, Mounkaila Noma ${ }^{1}$, \\ Mamoun Homeida ${ }^{1}$ \\ ${ }^{1}$ University of Medical Sciences and Technology, Sudan \\ *Corresponding author: Suha Mahir Atabani: amst33@hotmail.com
}

\section{Abstract:}

Citation: Atabani S.M., Khali S.I., Noma M., Homeida M. (2017) Prevalence and Clinical Presentations of Dilated Cardiomyopathy in Sudanese Patients with Heart Failure. Open Science Journal 2(4)

Received: $23^{\text {rd }}$ July 2017

Accepted: 10 ${ }^{\text {th }}$ October 2017

Published: $9^{\text {th }}$ December 2017

Copyright: (c) 2016 This is an open access article under the terms of the Creative Commons

Attribution License, which permits unrestricted use, distribution, and reproduction in any medium, provided the original author and source are credited.

Funding: The author(s) received no specific funding for this work

Competing Interests: The author have declared that no competing interests exists.

Background - Idiopathic dilated cardiomyopathy (DCM) is a heart muscle disease of undefined cause that commonly presents as congestive cardiac failure. The etiology of weakness of the heart muscle is often unknown, but many causal factors had been identified. The aim of this study was to determine the prevalence and clinical presentations of dilated cardiomyopathy in Sudanese patients with heart failure.

Method - A prospective hospital-based observational study was undertaken during the period of January to April 2007 in two teaching hospitals namely Academy Charity Teaching Hospital and Shaab Teaching Hospital. All patients diagnosed with heart failure were included in the study after the well informed of the patients and their treating physicians. A standardized questionnaire developed by the researcher was used to collect data on sociodemographic characteristics of patients, results of clinical, laboratory and radiography exams were recorded. All data were analyzed through the Statistical package for Social Sciences. Significance testing of difference between proportions was conducted using the Chi-square test were applicable, adjusted by Pearson's or Fisher's exact test, depending on the number of observations, with a value corresponding to $\mathrm{p}<0.05$ for significance unless otherwise.

Results - Of the 72 patients in the study, $53.0 \%$ were males and $47.0 \%$ were females. The prevalence of DCM was found to be $43.1 \%$ (31 out of 72 heart failure patients). The prevalence was higher in males than females with a prevalence of respectively $47.4 \%$ and $38.2 \%$. 
There was no significant difference in gender distribution of DCM $(\mathrm{p}=0.4)$. DCM affected older age with a mean age of 55.4 years $(\mathrm{SEM}=2.5)$. Palpitations, severe grade of dyspnea (grade 3 and 4$)$, raised jugular venous pressure and cardiomegaly were the most frequent clinical presentations. The mean ejection fraction in DCM was found to be $36.7(\mathrm{SEM}=1.4)$.

Conclusion - Contrary to common belief among clinicians in Sudan DCM is one of the commonest causes of heart failure. Its prevalence called up a best practice among clinicians, in particular those practicing in emergency units.

Keywords: Idiopathic dilated cardiomyopathy, Cardiac, Heart failure

\section{Introduction}

Cardiomyopathies are diseases of the myocardium associated with cardiac dysfunction [1]. There are five types of cardiomyopathy which are namely dilated, hypertrophic, restrictive, arrhythmogenic right ventricular, and unclassified cardiomyopathy. The World Health Organization (WHO) defines dilated cardiomyopathy as ventricular chamber exhibiting increased diastolic and systolic volume (left ventricular end-diastolic size $>115 \%$ of that calculated for age and body surface area) and a low $(<40 \%)$ ejection fraction [1-2]. According to the classification of WHO/International Society and Federation of Cardiology, dilated cardiomyopathy in its primary (idiopathic or familial) and secondary forms is the most common cause of the clinical syndrome of chronic heart failure [1]. All 4 cardiac chambers are dilated and are sometimes hypertrophied. Dilation is more pronounced than hypertrophy, and the left ventricle is most often affected than the right. The cardiac valves are intrinsically normal, although the mitral and tricuspid valve rings are dilated and the valve leaflets do not oppose each other in systole, resulting in mitral and/or tricuspid regurgitation. Persistent mitral regurgitation leads to thickening of the mitral valve leaflets, and, at times, it is difficult to distinguish this thickening from other causes of mitral regurgitation. Thrombus formation (secondary to the low-flow cardiac output state) is often seen in the left ventricular apex and, at times, in the atria. Occasionally, the right ventricle is preferentially involved in the cardiomyopathy process. In the United States, the reported incidence of dilated cardiomyopathy varies annually from approximately 5-8 cases per 100,000 population. However, this number is likely to be an underestimate, owing to underreporting or under detection of asymptomatic patients, which may occur in as many as $50-60 \%$. The age-adjusted prevalence in the United States averaged 36 cases per 100,000 population [3-5]. Internationally, in Western countries, $1-1.5 \%$ of the adult population has dilated cardiomyopathy [6]. In general, approximately one third of the patients die from the disease, another one third continues to have chronic heart failure requiring therapy, and a third of the patients experienced improvement of their condition. Its cost, disability, and morbidity are among the highest of any disease [7-9]. 
A study was carried out in Sweden to estimate the proportion of idiopathic dilated cardiomyopathy (IDCM) among congestive heart failure (CHF) patients, and to evaluate its prognostics. The records of all 16-65-year-old patients hospitalized for CHF or IDCM during a 6-year period $(\mathrm{n}=2711)$ were evaluated in a defined region of Western Sweden (1.05 million inhabitants aged 16-65 years ). Twenty-two percent $(584 / 2711)$ of these records contained no plausible causes of $\mathrm{CHF}$ or IDCM, and among living patients an obvious etiology was lacking in $27 \%$ (411/1516). The 411 patients went through further diagnosis including echocardiography. They, were compared to a randomly selected healthy control group $(\mathrm{n}=103)$. Of 411 patients, 293 participated in the echocardiographic investigations and 286 had acceptable echocardiographic recordings, indicating left ventricular dilatation and systolic dysfunction in 30\%. From the hospital records, 170 patients were identified as new cases of IDCM during the 6-year period. Adding another 34 cases revealed by diagnostic procedures yielded an agegender standardized incidence rate of 29.2 cases per $10(6)$ persons/year. The incidence of IDCM increased considerably with age, although in younger patients its relative contribution to heart failure was greater. The incidence of IDCM was higher in the urban compared to the rural parts of the region 21 versus $32 / 10(6)$; $\mathrm{P}=0.013)$. The estimated prevalence was 131/10(6) [10]. Estimates of the incidence of idiopathic dilated cardiomyopathy (IDCM) were obtained by identifying cases from Washington County, Maryland who were hospitalized at local and regional hospitals over a recent 17-year period. All of the verified cases had evidence of ventricular dilatation and hypokinesis, with a left ventricular ejection fraction of less than $40 \%$. A total of 26 cases were hospitalized over the period 1975-1991. The average age of the cases at the time of diagnosis was 55.5 years (range 22-80 years). About $58 \%$ of the subjects were males. A marked increase in newly diagnosed cases of IDCM occurred in this population in recent years; $38 \%$ of the cases (10 of 26) were newly diagnosed during 1991 alone. Over the period 1975-1991, the average annual incidence rate of hospitalized cases was 1.6 per 100,000 among men and 1.2 per 100,000 among women. Over the last 7 years of the case ascertainment period (1985-1991), the average annual incidence rate was 3.5 per 100,000 among men and 2.5 per 100,000 among women. These results provide evidence that IDCM is being increasingly recognized by clinicians, and underscore the need for an improved understanding of the distribution and determinants of this often fatal condition [11].

A cross sectional survey s conducted in Harare Central hospital in Zimbabwe aimed to determine the distribution of cardiac diseases admitted in a tertiary referral hospital. 1,507 patients referred for echocardiographic evaluation were included to measure the occurrence of different cardiac diagnoses. This study stated that $1,153(76.5 \%)$ echocardiographic findings were abnormal, while 354 were normal. Rheumatic heart disease was the predominant diagnosis $(25.1 \%)$ with $208(74.3 \%)$ of cases being females. The main valvar lesion in females was mitral stenosis $(48.1 \%)$, while in males it was mitral regurgitation (61.1\%). Other diagnoses were: pericardial disease 250 (22.4\%), dilated cardiomyopathy 245 $(22.0 \%)$, hypertensive heart disease 148 (13.3\%) and others (17.4\%). There were 65 cases of peripartum cardiomyopathy among the cases of dilated cardiomyopathy and 34 cases of acute myocarditis among others. They concluded that detailed clinical, radiological and echocardiographic assessments enabled a 
credible diagnosis.. Echocardiography is an appropriate technology in this setting given the range of "echo-friendly" cardiac lesions found [12].

In Sudan, five hundred and thirty-nine patients with cardiovascular disease (CVD) were admitted during the period of 1980 to 1983 , representing $5.9 \%$ of admissions to Khartoum North Hospital; $52.7 \%$ were females and $47.3 \%$ males. Hypertensive heart disease (HHD) contributed to $33.8 \%$ of the cardiac admissions, while $26.5 \%$ were rheumatic heart disease (RHD) and $8.7 \%$ were due to acute rheumatic fever (RF). $17.8 \%$ were due to coronary artery disease (CAD). Congenital heart disease (CHD) constituted $3.9 \%$ and cardiomyopathies represented $5.0 \%$ of CVD admissions. $68.6 \%$ of the patients were from urban residence while $31.2 \%$ were from suburban locality [13].

This article presents the prevalence and clinical presentations of dilated cardiomyopathy among patients with heart failure admitted in two hospitals of Khartoum, the capital city of Sudan.

\section{Method}

A prospective observational study design was conducted from January to April 2007 in two teaching hospitals (Academy Charity and Al-Shaab) of Khartoum. All patients diagnosed to have heart failure were included in the study after their consent and the one of their treating physician; causes or etiological factors were noted.

All patients with DCM were questioned for their age, tribe, origin, occupation and alcohol ingestion. They were examined for elicit pulse rate, its rhythm, volume, synchronicity and character as well as peripheral pulses. Blood pressure, signs and symptoms of heart failure such as dyspnea, raised jugular venous pressure, congestive hepatomegaly, bilateral basal lung crepitation and peripheral oedema were recorded. They were also investigated to obtain hemoglobin level, cardiac enzymes. Thyroid function test, liver function test, renal function test, chest x-ray, electrocardiography and echocardiography were performed.

Data were collected through a master sheet designed in MS Excel. The data was analyzed using statistical package of social sciences SPSS,. Significance testing of difference between proportions was conducted using the Chi-square test were applicable, adjusted by Pearson's or Fisher's exact test, depending on the number of observations, with a value corresponding to $\mathrm{p}<0.05$ for statistical significance.. Results are expressed as mean and standard error of mean. The significance of difference between mean values among case and control groups was determined by the Student independent $t$ test, with $\mathrm{p}<0.05$ considered significant. 


\section{Results}

\section{Prevalence of Dilated Cardiomyopathy in Heart failure patients}

The geographical origin of the seventy-two heart failure patients was predominantly the Northern (30.0\%), Western (22.0\%) and Central $(21.0 \%)$ regions. The remaining $15.0 \%$ ethnic origin were Central (8.0\%), Southern $(4.0 \%)$ and two patients was classified as others.

Regarding the source of income, $90.0 \%$ did not have regular income; they were housewives $(42.0 \%)$, non-skilled $(40.0 \%)$ and students $(8.0 \%)$. The remaining $10.0 \%$ with regular income were classified as professional (7.0\%) and skilled $(3.0 \%) .58 .1 \%$ resided in urban area and $41.9 \%$ lived in rural area. Males were more affected by heart failure than females with respectively a prevalence of $53.0 \%$ and $47.0 \%$. $57.7 \%$ of the heart failure patients were $\leq 55$ years and $42.3 \%$ were above 55 years old.

Of 72 cases of heart failure examined, 31 patients presented DCM leading to an overall prevalence of DCM of $43.0 \%$. This prevalence ranged from $38.2 \%$ among females with heart failure to $47.7 \%$ in males, however, the difference was found not to be statistically significant with a chi-square of 0.6105 and a p-value of 0.434 .

\section{Symptoms of Dilated Cardiomyopathy in Heart failure patients}

The symptoms of palpitations, orthopnea, paroxysmal nocturnal dyspnea (PND) and chest pain did not differ between patients of heart failure due to DCM or those with other causes. In heart failure patients with DCM $(n=31)$, the symptoms the most often reported (table 2) were palpitation (93.5\%), orthopnea $(80.6 \%)$, PND $(74.1 \%)$ and chest pain $(29.0 \%)$. The frequency of the symptoms revealed by patients of heart failure due to others causes $(n=41)$ were palpitations (95.1\%), orthopnea (73.1\%), PND (66.0\%) and chest pain $(32.0 \%)$. The grading of dyspnea revealed that in heart failure with DCM $(n=31)$, dyspnea was absent in one patient $(3.0 \%)$. In the remaining $97.0 \%$, dyspnea was observed on severe exertion (grade 1 dyspnea) in $7.0 \%$ of the patients, on moderate (grade 2 ) and mild exertion (grade 3 ) respectively $32.0 \%$ and $42.0 \%$ while dyspnea occurring at rest (grade 4$)$ was $16.0 \%$. In heart failure with other causes $(n=41)$, dyspnea was absent in two patients (5.0\%), the remaining 39 patients were classified as grade $2(44.0 \%)$, grade $3(41.0 \%)$ and grade $4(10.0 \%)$.

Table 1 compared DCM patients with other causes of heart failure. It revealed that except thyrotoxicosis, patients with heart failure due to DCM and those with heart failure due to other causes had almost the same background for hypertension, diabetes and alcohol consumption. However, any statistically significant association was not found between heart failure due to DCM and the history of hypertension $(\mathrm{p}=0.85791)$, diabetes $(\mathrm{p}=0.86141)$ and alcohol consumption $(\mathrm{p}=0.59458)$. 
Table 1: Symptoms and background history in patients with heart failure due to DCM and other causes

\section{Heart failure due to Heart failure due \\ other causes}

Heart failure

\section{Other}

\begin{tabular}{lcccccc} 
Variable & Yes & No & Yes & No & causes $\%$ & DCM \% \\
\hline Symptom & & & & & & \\
Palpitation & 39 & 2 & 29 & 2 & 95.1 & 93.5 \\
Orthopnea & 30 & 11 & 25 & 6 & 73.2 & 80.6 \\
Paroxysmal nocturne dyspnea & 27 & 14 & 23 & 8 & 65.9 & 74.2 \\
Chest pain & 13 & 28 & 9 & 22 & 31.7 & 29.0 \\
History of & & & & & & \\
Hypertension & 10 & 31 & 7 & 24 & 24.4 & 22.6 \\
Diabetes & 6 & 35 & 5 & 26 & 14.6 & 16.1 \\
Thyrotoxicosis & 1 & 40 & 0 & 31 & 2.4 & 0.0 \\
Alcohol consumption & 6 & 35 & 6 & 25 & 14.6 & 19.4 \\
\hline
\end{tabular}

\section{Clinical Presentations of Dilated Cardiomyopathy}

Patients with heart failure due to DCM were diagnosed with the condition for an average of 18.5 months \pm 4.6 . They were older (55.40 years \pm 2.5$)$ than heart failure due other causes patients; this difference was statistically different $(\mathrm{p}=0.03)$ as revealed by table 2 .

On examination of heart failure patients with DCM $(\mathrm{n}=31)$, the jugular venous pressure (JVP) was raised in $97.0 \%$ and $19.4 \%$ were pale. Their mean systolic blood pressure was found to be $126.77 \mathrm{~mm} \mathrm{Hg}(\mathrm{SEM}=3.12)$ and the diastolic to be $83.06 \mathrm{~mm} \mathrm{Hg}(\mathrm{SEM}=2.4)$, however any statistical difference was found both for the means systolic $(\mathrm{p}=0.78)$ and diastolic $(\mathrm{p}=0.09)$ blood pressure between the two groups of heart failures.48.4\% of DCM patients were found to have their apex beat in the 6th intercostal space anterior axillary line, $25.8 \%$ in the 5th intercostal space midclavicular line, $19.4 \%$ in the 6 th intercostal lateral to the midclavicular line while only $6.5 \%$ had it in the 5 th intercostal space just lateral to the midclavicular line. In other heart failure causes, the majority of patients $(80.0 \%)$ had an apex beat in the 5th intercostal space midclavicular line and only $12.5 \%$ in the 6th intercostal space anterior axillary line. When examining the character of the cardiac impulse, $87.1 \%$ of DCM patients were found to have a heaving impulse while only $36.6 \%$ of other heart failure patients had a heaving impulse.

Table 2: Summary of cardiovascular findings in heart failure patients due to DCM and other causes

\begin{tabular}{|c|c|c|c|}
\hline Variables & $D C M(n=31)$ & Others $(n=41)$ & $p$ value \\
\hline Mean Duration since diagnosis with DCM (SEM) & $18.5(4.6)$ & & \\
\hline Mean quantity of alcohol consumed per week (SEM) & $2666 \mathrm{ml}(882)$ & $1666 \mathrm{ml}(333)$ & 0.3 \\
\hline Mean Pulse rate (SEM) & 86.9 (2.3) & $88.6(2.4)$ & 0.60 \\
\hline
\end{tabular}




\begin{tabular}{llll}
\hline Mean Systolic BP (SEM) & $126.77(3.12)$ & $128.38(2.9)$ & 0.78 \\
Mean Diastolic BP (SEM) & $83.06(2.4)$ & $76.37(2.9)$ & 0.09 \\
Mean number of valves affected (SEM) & $1.5(0.15)$ & $1.1(0.19)$ & 0.11 \\
Mean Hb (SEM) & $81.15(1.4)$ & $77.55(2.3)$ & 0.22 \\
Mean left ventricular size (SEM) & $5.96(0.18)$ & $4.63(0.13)$ & 0.00 \\
Mean right ventricular size (SEM) & $5.41(0.19)$ & $4.09(1.9)$ & 0.00 \\
Mean Ejection fraction (SEM) & $36.7(1.4)$ & $43.4(1.5)$ & 0.00 \\
\hline
\end{tabular}

On palpation of pericardium for thrills, $80.6 \%$ of the 31 DCM patients demonstrated thrills in mitral area and in patients with heart failure due to other causes $(n=41)$, thrills were palpable in the mitral area for $41.5 \%$. The difference was statistically significant (chi-square $=11.50 ; \mathrm{p}=0.0008)$. In tricuspid area, thrills were present in $32.3 \%$ of the DCM patients and $7.3 \%$ (3 patients) of the heart failure cases due to other causes. No DCM patient had palpable in thrills in aortic area, thrills in aortic area were presents (19.5\%) in patients with heart failure due to other causes. Left parasternal heave was prevalent in DCM patients and other causes of heart failure for respectively $77.4 \%$ and $36.6 \%$ and the difference was statistically significant (chi-square $=11.8561 ; \mathrm{p}=0.0005$ ). In aortic area, thrills were noted in $19.5 \%$ of the heart failure due to others causes and was nil for the 31 DCM patients.

On auscultation, a normal first mitral heart sound was recorded in $19.4 \%$ of DCM patients and it was present in $48.8 \%$ of the patients with heart failure due to other cause. This difference was statistically significant with a chi-square of 6.625 and a p-value of 0.010 . A muffled first mitral heart sound was present in $80.6 \%$ of the DCM patients and $19.5 \%$ of those with heart failure due to other causes. The difference was statistically significant (chi-square $=26.576 ; \mathrm{p}=0.000$ ). A loud first mitral heart sound was not diagnosed in DCM patients, but it was present in $31.7 \%$ of the heart failure due to other causes. A normal first tricuspid heart sound was recorded was respectively $35.5 \%$ and $90.2 \%$ in DCM patients $(n=31)$ and heart failure due to other causes $(n=41) .64 .5 \%$ of the DCM patients had muffled first tricuspid heart sound; its prevalence in patients with heart failure due to other causes was $7.3 \%$. Loud first tricuspid heart sound was recorded in none of the DCM patients, it was present in $2.4 \%$ in those with heart failure due to other causes. The second aortic heart sound was normal in both DCM patients $(96.8 \%)$ and heart failure due to other causes $(80.5 \%)$. It was muffled in DCM patients and in those with other causes respectively $3.2 \%$ and $12.2 \%$. Loud second aortic heart sound was not recorded in DCM patients; it was prevalent in $7.3 \%$ of the heart failure due to other causes.

The pulmonary second heart sound was found to be normal in all patients with DCM in contrast to patients with heart failure due other causes for whom normal, loud and muffled sound were respectively $53.7 \%, 43.9 \%$ and $2.4 \%$.

Concerning the valvar lesions, any valvar lesion was noted in $12.9 \%$ of the DCM patients and in $39.0 \%$ of the patient with other causes of heart failure. All the valves were affected in patients with other causes of heart failure (table 3), DCM patients had one (29.0\%), two (51.6\%) and three (6.5\%) valve lesions. When comparing DCM patients with two valves lesions (51.6\%) with those with 
heart failure due to other causes and having the same number of valves lesions $(22.0 \%)$, a statistically significant difference was found with chi-square of 6.8522 and a p-value of 0.00885 .

The signs of heart failure in DCM patients $(n=31)$ were hepatomegaly $(87.1 \%)$, bilateral basal crepitation $(90.3 \%)$ and lower limb edema (93.5\%) while in patients of heart failure due to other causes hepatomegaly, bilateral basal crepitation and lower limb edema were respectively $80.5 \%, 97.6 \%$ and $65.9 \%$.

Table 3: Clinical presentation of Dilated Cardiomegaly and heart failure due to other causes patients

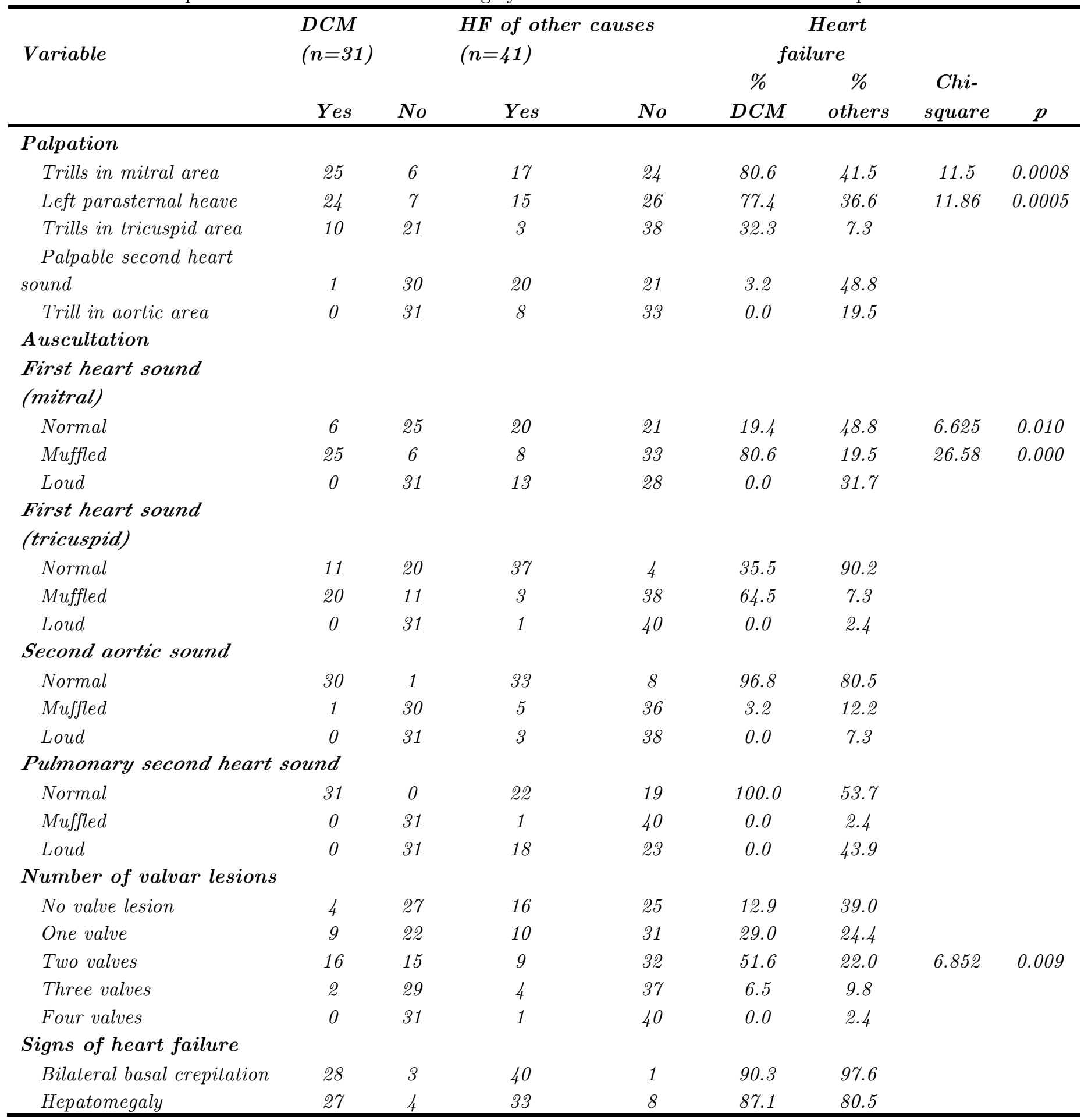




\begin{tabular}{|c|c|c|c|c|c|c|c|c|}
\hline Lower limb edema & 29 & 2 & 27 & 14 & 93.5 & 65.9 & & \\
\hline \multicolumn{9}{|c|}{ Chest $X$-ray findings } \\
\hline \multicolumn{9}{|l|}{ Cardiomegaly } \\
\hline Cardiomegaly & 30 & 1 & 20 & 21 & 96.8 & 48.8 & & \\
\hline No cardiomegaly & 1 & 30 & 21 & 20 & 3.2 & 51.2 & & \\
\hline \multicolumn{9}{|c|}{ Lung field on chest $x$-ray } \\
\hline Normal & 3 & 28 & 19 & 22 & 9.7 & 46.3 & & \\
\hline Oligaemic & 1 & 30 & 0 & 41 & 3.2 & 0.0 & & \\
\hline Plethoric & 27 & 4 & 22 & 19 & 87.1 & 53.7 & & \\
\hline \multicolumn{9}{|l|}{$E C G$ and } \\
\hline \multicolumn{9}{|l|}{ Echocardiography } \\
\hline \multicolumn{9}{|l|}{ Rhythm on ECG } \\
\hline Sinus rhythm & 22 & 9 & 25 & 16 & 71.0 & 61.0 & 0.778 & 0.378 \\
\hline Artrial fibrillation & 6 & 25 & 13 & 28 & 19.4 & 31.7 & 1.387 & 0.239 \\
\hline Venal ectopic & 1 & 30 & 0 & 41 & 3.2 & 0.0 & & \\
\hline others & 2 & 29 & 3 & 38 & 6.5 & 7.3 & & \\
\hline \multicolumn{9}{|c|}{ Ventricular hypertrophy } \\
\hline Present & 29 & 2 & 19 & 22 & 93.5 & 46.3 & & \\
\hline Absent & 2 & 29 & 22 & 19 & 6.5 & 53.7 & & \\
\hline \multicolumn{9}{|l|}{ Artrial hypertrophy } \\
\hline Present & 7 & 24 & 16 & 25 & 22.6 & 39.0 & 2.196 & 0.138 \\
\hline Absent & 24 & 7 & 25 & 16 & 77.4 & 61.0 & & \\
\hline \multicolumn{9}{|c|}{ Identification of clots } \\
\hline Present & 2 & 29 & 6 & 35 & 6.5 & 14.6 & & \\
\hline Absent & 29 & 2 & 35 & 6 & 93.5 & 85.4 & & \\
\hline \multicolumn{9}{|c|}{ Thyroid function test } \\
\hline Low TSH & 1 & 30 & 3 & 38 & 3.2 & 7.3 & & \\
\hline High T3 & 1 & 30 & 3 & 38 & 3.2 & 7.3 & & \\
\hline High T4 & 1 & 30 & 3 & 38 & 3.2 & 7.3 & & \\
\hline
\end{tabular}

Chest X-ray revealed that $96.8 \%$ of the DCM patients had cardiomegaly while one $(3.2 \%)$ did not have it. In the 41 heart failure patients due to other causes, cardiomegaly was present in $48.8 \%$ and absent in $51.2 \%$. The lung appeared to be plethoric in $87.1 \%$ of the DCM and in $53.7 \%$ of the patients with heart failure to other causes.

Electro-cardiogram and Echocardiography findings, sinus rhythm (71.0\%) and atrial fibrillation (19.4\%) were the most frequent ECG findings in DCM patients. In heart failure patients due to other causes, sinus rhythm and atrial fibrillation were respectively $61.0 \%$ and $31.7 \%$. For sinus rhythm, any statistical difference was found between DCM patients and those with heart failure due to other causes (chi-square $=0.7776 ; \mathrm{p}=0.3778$ ). The difference regarding atrial fibrillation between DCM patients and those with other causes of heart failure was also not statistically significant (chi-square $=1.3866 ; \mathrm{p}=0.2389$ ).

Ventricular hypertrophy was present in $93.5 \%$ of the 31 DCM patients and absent in two (6.5\%), the condition was recorded present and absent respectively in $46.3 \%$ and $53.7 \%$ of the heart failure due to other causes $(n=41)$. The mean left ventricular size was found to be 5.96 (SEM: 0.18) in DCM patients and 4.63 (SEM: 0.13) in other heart failure patients. For the right 
ventricular, mean size (was found to be 5.41 (SEM: 0.19) and 4.09 (SEM:1.9) in DCM and other heart failure patients respectively. Table 2 revealed that there was a statistically significant difference when comparing DCM and heart failure due to other causes. The mean ejection fraction was estimated to be 36.7 (SEM: 1.4) in DCM patients which was statistically significant when judged against the 43.4 (SEM: 1.5) of the other heart failure patients (table 2).

Atrial hypertrophy was found in $22.6 \%$ of DCM patients and $39.0 \%$ of the heart failure due to other causes. The difference was not statistically significant $(\mathrm{p}=0.1384)$. Clots were visualized in $6.5 \%$ of DCM patients and $14.6 \%$ of heart failure due to other causes.

Thyroid function test results in DCM patients were identical for all the levels and similar distribution was recorded in heart failure due to other causes (table 3).

Treatment and evolution, the most frequent treatment received by DCM and heart failure due to other causes patients were diuretics $(96.8 \%$ of DCM patients, $100 \%$ heart failure due to other causes), ACEI $(90.3 \% ; 78.0 \%)$ and Spironolactone $(64.5 \% ; 78.0 \%)$. Aspirin and Digoxin were administrated respectively $32.3 \%$ and $19.4 \%$ of the DCM patients. Heart failure due to other treated with aspirin and digoxin were respectively $19.5 \%$ and $41.5 \%$. Under these treatments the evolution of condition of DCM patients were improving $(80.6 \%)$, statistic $(6.5 \%)$ and deteriorating $(6.5 \%)$.

Etiology of DCM, of the 31 DCM patients the etiology was unknown for the majority $(71.0 \%)$, the remaining $29.0 \%$ DCM the etiology was ischemic $(19.4 \%)$, Puerperal $(6.5 \%)$ and Thyrotoxic $(3.2 \%)$.

\section{Conclusions}

The research findings revealed that Dilated Cardiomyopathy (DCM) prevailed in Sudanese population with heart failure. It had an overall prevalence of $43.0 \%$ (31 DCM out 72 heart failure). This prevalence was higher than the $5 \%$ prevalence DCM reported out of 539 patients with cardiovascular disease (CVD) admitted during the period of 1980 to 1983 in Khartoum North hospital [13]. Rather than claiming an increase of the condition between this study and ours, we plead for heightened awareness among health professional of the significant cardiac morbidity to attempt to understand the epidemiologic factors within heart failure patients and to enhance the diagnostic of DCM. Elsewhere in Africa a prevalence rate of DCM of $22 \%$ was given in a study conducted in Zimbabwe [12].

The prevalence of DCM among males with heart failure was not significantly different when compared to that among females in our research; however, men being 3 folds more involved than women was stated in a study done in the United States [14], and men being affected twice as commonly as women was declared in a meta-analysis done in Africa [15].

Regarding the age of occurrence of DCM, it affected more the older age $(55.4$ years \pm 2.5 ) than patients with heart failure due to other causes (47.1 years $\pm 2.9)$ with a statistically significant difference $(\mathrm{p}=0.03)$. 
The most common symptoms in both DCM and heart failure due others causes were palpitations, dyspnea, orthopnea, paroxysmal nocturnal dyspnea and chest pain.

These symptoms did not differ significantly between male and female patients. Neither did they differ between the age groups. However, it was noticed that all patients older than 55 years of age complained of palpitations and the percentage of them having chest pain was greater than that of the younger age group.

Regarding the causes of DCM, it was estimated that $71 \%$ of DCM were of unknown cause. This is comparable to the $50 \%$ of idiopathic DCM given in a study done in the United States [14].

Physician awareness of this condition must be enhanced, this will in turn lead to both patient and institutional benefits. If the DCM can be diagnosed early, manifestations of the condition maybe controlled or even prevented giving the patient a longer and better quality of life. For governmental or private institutions, , proper diagnosis would mean less hospital stay for the affected and less strain on the already exhausted staff in addition to the financial benefits..

Patient education is an effective simple tool, unfortunately not being properly used in Sudan. While the patient is in reach, he should not only be diagnosed but also educated on his condition, on how they acquired it and how they can control it. The risk factors should be made known to them and also the warning signs of heart failure. Further to this, they should be advised to enlighten all aging close contacts whom may also be at risk of the early symptomatology of this rather serious disease.

\section{List of abbreviations}

CAD Coronary artery disease

CVD Cardiovascular disease

CHD Congenital heart disease

CHF Congestive heart failure

DCM Idiopathic dilated cardiomyopathy

HHD Hypertensive heart disease

PND Paroxysmal nocturnal dyspnea

JVP Jugular venous pressure

RF Acute rheumatic fever

RHD Rheumatic heart disease

SEM Standard error of the mean

\section{Authors' contributions}

SMA collected and analyzed the data. SIK and MH conceived and designed the research. MN drafted the manuscript which was read and approved by all the authors. 


\section{References:}

1. Richardson P, McKenna W, Bristow M, et al. Report of the 1995 World Health Organization/International Society and Federation of Cardiology Task Force on the Definition and Classification of cardiomyopathies. Circulation. 1996; 93:841-42.

2. Boffa GM, Thiene G, Nava A, Dalla Volta S: Cardiomyopathy: a necessary revision of the WHO classification. Int J Cardiol 1991 Jan; 30(1): 1-7.

3. Gillum RF: Idiopathic cardiomyopathy in the United States, 1970-1982. Am Heart J 1986 Apr; 111(4): 752-5

4. Codd MB, Sugrue DD, Gersh BJ: Epidemiology of idiopathic dilated and hypertrophic cardiomyopathy. A population-based study in Olmsted County, Minnesota, 1975-1984. Circulation 1989 Sep; 80(3): 564-72.

5. Manolio TA, Baughman KL, Rodeheffer R: Prevalence and etiology of idiopathic dilated cardiomyopathy (summary of a National Heart, Lung, and Blood Institute workshop. Am J Cardiol 1992 Jun 1; 69(17): 1458-66.

6. $\quad$ eMedicine: H R Afridi: Dilated cardiomyopathy: Feb 2007.

7. Ho KK, Anderson KM, Kannel WB, et al: Survival after the onset of congestive heart failure in Framingham Heart Study subjects. Circulation 1993 Jul; 88(1): 107-15.

8. O'Connell JB, Bristow MR: Economic impact of heart failure in the United States: time for a different approach. J Heart Lung Transplant 1994 Jul-Aug; 13(4): S107-12.

9. Guccione AA, Felson DT, Anderson JJ, et al: The effects of specific medical conditions on the functional limitations of elders in the Framingham Study. Am J Public Health 1994 Mar; 84(3): 351-8.

10. Andersson B, Caidahl K, Waagstein F: Idiopathic dilated cardiomyopathy among Swedish patients with congestive heart failure. Eur Heart J. 1995 Jan;16(1):53-60.

11. Coughlin SS, Comstock GW, Baughman KL: Descriptive epidemiology of idiopathic dilated cardiomyopathy in Washington County, Maryland, 1975-1991. J Clin Epidemiol. 1993 Sep;46(9):1003-8.

12. Hakim JG, Manyemba J: Cardiac disease distribution among patients referred for echocardiography in Harare, Zimbabwe. Cent Afr J Med. 1998 Jun; 44(6):140-4.

13. S I Khalil, E Z Elsamani, G Dafalla, E Kazzam, A Rabeh: Patterns of cardiovascular disease in Sudan: Hospital load and recent trends. Sudan Med. J. 1984, vol. 20; 4:25-38.

14. Olbrich HG: Epidemiology-etiology of dilated cardiomyopathy. Z Kardiol. 2001;90 Suppl 1:2-9.

15. Karen Sliwa, Albertino Damasceno: Epidemiology and Etiology of Cardiomyopathy in Africa. Circulation. 2005; 112:3577-3583. 